\title{
Human chorionic gonadotropin therapy in hypogonadic severe-oligozoospermic men and its effect on semen parameters
}

\author{
Syed Waseem Andrabi ${ }^{1}$, Giresh Chandra Makker ${ }^{2}$, Renu Makker ${ }^{2}$, Geetanjali Mishra ${ }^{1}$, Rajender Singh ${ }^{3}$ \\ 'Department of Zoology, University of Lucknow, Lucknow; ${ }^{2}$ Makker IVF Centre, Lucknow; ${ }^{3}$ Division of Endocrinology, CSIR-Central Drug Research \\ Institute, Lucknow, India
}

Objective: This study aimed to evaluate whether human chorionic gonadotropin ( $\mathrm{hCG}$ ) therapy is beneficial for improving semen parameters and clinical hypogonadism symptoms in hypogonadic oligozoospermic or severe oligozoospermic men with low or borderline testosterone levels.

Methods: A weekly dose of $250 \mu \mathrm{g}$ (equivalent to approximately 6,500 IU) of hCG was administered subcutaneously for 3-6 months to 56 hypogonadic oligozoospermic or severe oligozoospermic men. Semen, biochemical, and genetic analyses were performed before the start of treatment followed by analyzing semen parameters every 3 months after the start of therapy. We grouped participants into responders and non-responders depending on positive changes in semen parameters.

Results: Out of 56 men, $47(83.93 \%)$ responded, while 9 (16.07\%) did not. Upon statistical analysis, it was found that age did not affect the overall outcomes $(p=0.292)$; however, men with higher body mass index $\left(\mathrm{BMl} ; 28.09 \pm 3.48 \mathrm{~kg} / \mathrm{m}^{2}\right)$ showed better outcomes than those with low BMI $\left(25.33 \pm 3.06 \mathrm{~kg} / \mathrm{m}^{2}\right)(p=0.042)$. The duration of therapy (in months) was higher in non-responders than in responders $(p=0.020)$. We found significant improvements in sperm concentration $(p=0.006)$ and count $(p=0.005)$ after 3 months of therapy. Sperm motility and progressive motility were also found to be higher in responders, but did not show statistically significant changes.

Conclusion: We conclude that hCG therapy can be beneficial in men with hypogonadic oligozoospermia or severe oligozoospermia.

Keywords: Human chorionic gonadotropin; Hypogonadism; Oligozoospermia

\section{Introduction}

Male and female factors contribute equally to about $15 \%$ of couples with fertility issues [1]. Men with hypogonadism have diminished levels of testosterone, and hypogonadism coupled with re-

Received: June 7, 2021 · Revised: October 18, 2021 · Accepted: November 13, 2021 Corresponding author: Giresh Chandra Makker

Makker IVF Centre, 3c, Tilak Marg, Hazratganj, Lucknow, Uttar Pradesh 226001, India Tel:+91-522-4008289 E-mail:makkermed@gmail.com

Co-Corresponding author: Rajender Singh

Division of Endocrinology, CSIR-Central Drug Research Institute, Sector10, Jankipuram Extension, Sitapur Road, Lucknow, Uttar Pradesh 226031, India Tel: +91-522-2612411-18 Fax:+91-522-22771941 E-mail: rajender_singh@cdri.res.in

*This study was funded by Council of Scientific and Industrial Research (CSIR) under MLP0113-DeMID to Rajender Singh.

This is an Open Access article distributed under the terms of the Creative Commons Attribution Non-Commercial License (http://creativecommons.org/licenses/by-nc/4.0/) which permits unrestricted non-commercial use, distribution, and reproduction in any medium, provided the original work is properly cited. duced gonadotropin levels (luteinizing hormone $[\mathrm{LH}]$ and follicle-stimulating hormone [FSH]) is known as hypogonadic hypogonadism $(\mathrm{HH})$. $\mathrm{HH}$ can either be congenital or acquired. The classical example of congenital hypogonadism is Kallmann syndrome, while acquired hypogonadism can occur due to pituitary lesions causing hyperprolactinemia or as a result of anabolic steroid usage. During spermatogenesis, which is regulated by hormones, the differentiation of stem cells starts in the testes. Gonadotropin-releasing hormone $(\mathrm{GnRH})$ from the hypothalamus is released in a pulsatile manner to stimulate the production of FSH and $\mathrm{LH}$ in the anterior lobe of the pituitary gland [2]. FSH helps in spermatogenesis by directly stimulating Sertoli cells, while LH stimulates testosterone production from Leydig cells. Testicular testosterone regulates spermatogenesis by its action on Sertoli cells, while peripheral testosterone plays an important role in sexual function in men in the form of libido, erectile function, and ejaculation [3]. For successful spermatogenesis, the 
functioning of the hypothalamus-pituitary-gonadal axis is critical and any alterations in this axis can give rise to hypogonadism, which can result in spermatogenic failure and affect male fertility [4].

Generally, testosterone levels start declining after around 40 years, and by 80 years, they are reduced by almost half [5]. Hypogonadism is characterized by low serum testosterone levels coupled with low libido, erectile dysfunction, low energy, depression, anemia, infertility, and many more clinical symptoms [6-8]. Hypogonadism affects $38 \%$ of men over 45 years of age and $7 \%$ of men under 40 years of age $[9,10]$. Although the clinical symptoms of hypogonadism are primarily signs of aging in men [6], early-onset hypogonadism may also affect fertility in men of reproductive age. In the past few decades, its prevalence in younger generations has increased exponentially, probably due to the use of anabolic-androgenic steroids [9]. Human chorionic gonadotropin (hCG), an analogue of LH that has a longer half-life (36 hours), plays an important role in the treatment of endocrine testicular failures and stimulates testosterone production by acting on Leydig cells [9]. In this study, hCG was administered to 56 hypogonadic infertile men with oligozoospermia with the goal of improving their semen profile.

\section{Methods}

This study was approved by the Institutional Ethics Committee of the Central Drug Research Institute, Lucknow (CDRI/IEC/2015/A1). The experiments were carried in accordance with the guidelines approved for research on human samples. Written informed consent was obtained from patients for publication of the submitted article and any accompanying images. Signed copies of the consent form were provided.

In total, 104 infertile men belonging to ethnic groups speaking Indo-Aryan languages who had idiopathic severe oligozoospermia (sperm concentration $>5$ million $/ \mathrm{mL}$ ) and hypogonadism symptoms were recruited at an infertility clinic between January 2014 to September 2019. All patients had completed puberty normally and had well-developed secondary sexual characteristics. A physical examination of testicular size was done by a well-trained urologist, and only patients with normal testicular sizes were included in this study. Men with a history of surgical intervention of the genital tract (e.g., for obstructive azoospermia or varicocele), drug abuse, radiation exposure, excessive alcohol consumption, abnormal karyotypes, and mental illness were excluded from the study. Out of 104 men, 25 were excluded due to a history of other treatments, while 18 other men were excluded due to incomplete hormonal therapy data. Five patients were excluded as they had Y-chromosome microdeletions, leaving 56 patients in this study. These patients had hypogonadism symptoms with initial total testosterone levels $<400 \mathrm{ng} / \mathrm{dL}$, imply- ing borderline testosterone levels. Informed written consent was obtained from all participants. Infertility in these men was defined as an inability to conceive after unprotected intercourse for more than 1 year. Female factor infertility was ruled out after a proper evaluation with a proper medical history of infertility treatment, as well as a general examination of the pelvic region and transvaginal ultrasonography showing no abnormality. All participants had primary infertility, and a detailed history including alcohol intake, smoking, family fertility history, lifestyle, libido, spontaneous erections, and anosmia was taken at their first visit.

Semen samples after 3-5 days of abstinence were collected by masturbation in a sterile container. Semen analysis was performed according to the 2010 World Health Organization guidelines [11]. The samples were allowed to liquefy and semen parameters were recorded. Oligozoospermia was confirmed by at least three semen analyses suggesting a below-normal sperm count.

\section{Genetic analysis}

To rule out genetic causes, karyotype and Y-chromosome microdeletion analyses were performed. Karyotype analysis confirmed a normal chromosomal complement $(46, \mathrm{XY})$ in all men. Y-chromosome microdeletions were analyzed using six STS markers for the AZFa (sY86 and sY84), AZFb (sY127, and sY134) and AZFc (sY255 and sY254) regions according to the recommendations of the European Academy of Andrology and the European Quality Monitoring Network Group [12]. Five men with Y-chromosome microdeletions were excluded from the study.

\section{Hormonal therapy}

Based on the testosterone levels (low or borderline) and clinical symptoms, a diagnosis of adult-onset hypogonadism ( $\mathrm{AOH}$ ) was made. After diagnosing, the male partners were suggested to receive hCG therapy at a weekly dose of $250 \mu \mathrm{g}$. During the treatment, semen analysis was performed after every 4 weeks. No sperm was found in the ejaculate samples even after centrifugation for the first 2 months in any participants. However, after the third month, occasional sperm were found in the ejaculate in two men and at the end of 4 months, six other men responded by showing occasional sperm. All the responders agreed to continue the therapy until 6 months and three men showed normal semen parameters in the ejaculate, while three men showed oligozoospermic parameters. As advised, the couples had regular sexual intercourse.

\section{Results}

In this single-center study, the patients were treated by a single urologist with specific training in male infertility. A total of 56 men 
met the inclusion criteria, all of whom received hCG therapy, followed by a classification of the patients into responders and non-responders depending upon the effect of hCG therapy on semen parameters. In responders, the sperm concentration was found to increase in the ejaculate, either during or after hCG therapy. In non-responders, no change in sperm concentration was found in the ejaculate post-treatment. In total, 47 men (83.93\%) responded and showed an increase in sperm concentration, while nine men (16.07\%) did not show any improvement in their semen parameters. The majority (57.1\%) of the men were between 31 and 40 years of age.

Upon comparing the age between two groups, the mean \pm standard deviation age of responders ( $32.00 \pm 4.31$ years) and non-responders (30.40 \pm 3.27 years) was not significantly different $(p=0.292)$. Unexpectedly, the body mass index was significantly higher $(p=0.042)$ in responders $\left(28.09 \pm 3.48 \mathrm{~kg} / \mathrm{m}^{2}\right)$ than in non-responders $\left(25.33 \pm 3.06 \mathrm{~kg} / \mathrm{m}^{2}\right)$. Upon comparing the duration of therapy, non-responders had a significantly longer $(p=0.020)$ duration than responders ( $4.74 \pm 1.03$ vs. $3.77 \pm 0.90$ months). No significant difference in the length of marriage was found $(p=0.895)$ between responders $(6.98 \pm 4.22$ years) and non-responders $(6.78 \pm 3.70$ years). Selected clinical characteristics of responders and non-responders are given in Table 1.

We also compared the results of pre-treatment hormonal analyses

Table 1. Comparison of clinical characteristics between responders and non-responders

\begin{tabular}{|c|c|c|c|}
\hline \multirow{2}{*}{ Parameter } & \multicolumn{2}{|c|}{ Outcome } & \multirow{2}{*}{$p$-value } \\
\hline & Responder & Non-responder & \\
\hline Age (yr) & $32.00 \pm 4.31$ & $30.40 \pm 3.27$ & 0.292 \\
\hline Duration of therapy (mo) & $3.77 \pm 0.90$ & $4.74 \pm 1.03$ & 0.020 \\
\hline Body mass index $\left(\mathrm{kg} / \mathrm{m}^{2}\right)$ & $28.09 \pm 3.48$ & $25.33 \pm 3.06$ & 0.042 \\
\hline Length of marriage (yr) & $6.98 \pm 4.22$ & $6.78 \pm 3.70$ & 0.895 \\
\hline
\end{tabular}

Values are presented as mean \pm standard deviation.

Table 2. Pre-treatment hormonal comparison between responders and non-responders

\begin{tabular}{|c|c|c|c|}
\hline \multirow{2}{*}{ Hormone } & \multicolumn{2}{|c|}{ Outcome } & \multirow{2}{*}{$p$-value } \\
\hline & Responder & Non-responder & \\
\hline $\mathrm{LH}(\mathrm{mlU} / \mathrm{mL})$ & $6.58 \pm 3.63$ & $3.88 \pm 2.14$ & 0.026 \\
\hline $\mathrm{FSH}(\mathrm{mIU} / \mathrm{mL})$ & $5.71 \pm 2.53$ & $7.38 \pm 4.21$ & 0.256 \\
\hline Total testosterone $(\mathrm{ng} / \mathrm{mL})$ & $3.52 \pm 0.68$ & $3.38 \pm 0.85$ & 0.639 \\
\hline Free testosterone $(\mathrm{pg} / \mathrm{mL})$ & $15.41 \pm 7.90$ & $8.47 \pm 4.48$ & 0.009 \\
\hline Prolactin $(\mathrm{ng} / \mathrm{mL})$ & $14.42 \pm 6.01$ & $8.33 \pm 3.96$ & 0.005 \\
\hline Estradiol (pg/mL) & $35.04 \pm 15.41$ & $34.76 \pm 15.59$ & 0.964 \\
\hline
\end{tabular}

Values are presented as mean \pm standard deviation.

LH, luteinizing hormone; $\mathrm{FSH}$, follicle-stimulating hormone. between the responders and non-responders. LH, free testosterone, and prolactin levels were significantly higher in responders than in non-responders (Table 2). However, no significant difference was observed in $\mathrm{FSH}$, total testosterone, and estradiol levels between responders and non-responders (Table 2).

The main target of hCG therapy was its effect on semen parameters. In the final observations of the two groups post-therapy, we found significant improvements in sperm concentration $(p=0.006)$ and the total sperm count $(p=0.005)$; however, motility and progressive motility improved, but the changes were not statistically significant (Table 3).

As a further analysis of the sperm concentration distribution preand post-therapy, we performed the paired-proportion test and found a significant increase ( $p=0.012$ ) of $25 \%$ in sperm concentration and a significant increase $(p=0.019)$ of $30.4 \%$ in the overall count (Table 4).

\section{Discussion}

In infertile men, it is important to diagnose the underlying cause of infertility in order to determine an appropriate fertility treatment. In infertile azoospermic and oligozoospermic men with $\mathrm{AOH}$, sperm restoration can be performed using pulsatile GnRH therapy, which

Table 3. Effects of hCG therapy on semen parameters of pre- and post-therapy

\begin{tabular}{lccc}
\hline Variable & Pre-therapy & Post-therapy & $p$-value \\
\hline Concentration (million/mL) & $3.03 \pm 3.25$ & $6.54 \pm 9.40$ & 0.006 \\
Count (million) & $6.57 \pm 4.26$ & $15.82 \pm 24.97$ & 0.005 \\
Motility (\%) & $46.71 \pm 23.37$ & $52.59 \pm 17.08$ & 0.067 \\
Progressive motility (\%) & $21.21 \pm 17.07$ & $23.95 \pm 16.11$ & 0.340 \\
\hline
\end{tabular}

Values are presented as mean \pm standard deviation. hCG, human chorionic gonadotropin.

Table 4. Sperm concentration and count distribution of pre- and post-hCG treatment

\begin{tabular}{lccc}
\hline Variable & Pre-treatment & Post-treatment & \% Difference \\
\hline $\begin{array}{l}\text { Concentration } \\
\leq 3 \text { million } / \mathrm{mL}\end{array}$ & $43(76.8)$ & $29(51.8)$ & -25.0 \\
$>3$ million/mL & $13(23.2)$ & $27(48.2)$ & 0.25 \\
$\quad$ Total & $56(100)$ & $56(100)$ & - \\
Count & & & \\
$\quad \leq 6$ million & $30(53.6)$ & $13(23.2)$ & -30.4 \\
$>6$ million & $26(46.4)$ & $43(76.8)$ & 0.304 \\
Total & $56(100)$ & $56(100)$ & - \\
\hline
\end{tabular}

Values are presented as number (\%). hCG, human chorionic gonadotropin. 
requires special equipment and is expensive compared to the simpler option of self- injectable gonadotropin therapy [13]. Gonadotropin therapy has also been reported to be more efficient than clomiphene citrate therapy [14].

The European Academy of Andrology suggested that when total testosterone levels are less than $350 \mathrm{ng} / \mathrm{dL}$, the likelihood of hypogonadism is high. However, lower testosterone levels below the reference range indicate a higher likelihood of testosterone deficiency. In patients with borderline testosterone levels, free testosterone can be used to establish a clear diagnosis. There are limited options for treating men with hypogonadism, and the most successful method is testosterone replacement therapy (TRT) [6]. TRT is also effective for promoting muscle strength energy, bone mineral density, and improvements in sexual function and vitality [8]. From 2001 to 2011, TRT has become four times as common among men between 40 and 49 years of age and three times as common overall [15]. hCG can be beneficial for men requiring TRT, and over the past decade, the importance of hCG has grown exponentially due to its usage in hypogonadic men.

hCG can be a promising alternative in men with $\mathrm{AOH}$. As an analogue of $\mathrm{LH}$, hCG stimulates the production and release of intratesticular testosterone by acting on Leydig cells, with the added benefit of a better half-life and self-administration (subcutaneously) [8]. Various protocols have been used in men with hypogonadism to induce and maintain spermatogenesis. Monotherapy of hCG or in combination with human menopausal gonadotropin can be given to azoospermic males for 3-6 months to achieve spermatogenic restoration, as shown by the presence or increase of spermatozoa in the ejaculate [16]. In our case, monotherapy of $250 \mu \mathrm{g}$ of hCG was successfully used to increase the sperm concentration in severely oligozoospermic men.

Furthermore, upon comparing pre-treatment hormonal profiles between responders and non-responders, we found that free testosterone was predictive of individuals' responses to hCG therapy, suggesting that along with testosterone, free testosterone levels should be also checked for diagnostic purposes in all patients before starting treatment. Testicular function improvements in the form of seminal parameters can be due to the beneficial effect of hCG therapy on Leydig and Sertoli cells. In our study, clinically modest response rates in sperm parameters (concentration and count) were observed, which could also be meaningful in further decision-making regarding various treatment options, including intrauterine insemination, in vitro fertilization, and intracytoplasmic sperm injection (ICSI).

Our results showed a positive response to therapy, indicating that the duration of treatment should be at least 3 months. Upon a further analysis of our results regarding the effect of hCG therapy on semen parameters, we found statistically significant improvements in semen concentration from $3.03 \pm 3.25$ million $/ \mathrm{mL}$ at baseline to $6.54 \pm 9.40$ million $/ \mathrm{mL}$ on average after 3 months of therapy $(p=0.006)$. Gonadotropic administration in infertile men with either azoospermia or oligozoospermia with high levels of FSH is usually useless. Hormonal therapy treatments in men with hypogonadic oligozoospermic have been overshadowed due to the use of ICSI, testicular sperm extraction (TESE), and micro-TESE. In men with hypogonadism, hCG therapy may be an option for spermatogenetic restoration when fertility is desired. Our data suggest that hCG therapy is safe and effective as an alternative to exogenous testosterone therapy. In a direct evaluation of the effects of hCG on semen parameters, our results showed significant improvements in semen concentration and count. The only challenge patients face is financial, as hCG therapy is costly; however, most of the time, the desire to achieve parenthood overcomes this barrier. In men with low or borderline testosterone levels, hCG therapy should be discussed as an option to retain fertility.

\section{Conflict of interest}

No potential conflict of interest relevant to this article was reported.

\section{Acknowledgments}

Rajender Singh is thankful to the Council of Scientific and Industrial Research (CSIR) for funding under MLP0113-DeMID.

\section{ORCID}

Syed Waseem Andrabi https://orcid.org/0000-0001-8749-9016

Giresh Chandra Makker https://orcid.org/0000-0002-9193-0558

Renu Makker

https://orcid.org/0000-0001-8720-8987

Geetanjali Mishra

https://orcid.org/0000-0001-8976-4602

Rajender Singh

\section{Author contributions}

Conceptualization: RS. Data curation: SWA. Formal analysis: GCM, RM. Funding acquisition: RS. Methodology: SWA, GM. Project administration: RS. Visualization: RS. Writing-original draft: SWA. Writingreview \& editing: RS, GM.

\section{References}

1. Waseem AS, Singh V, Makker GC, Trivedi S, Mishra G, Singh K, et al. AZF deletions in Indian populations: original study and me- 
ta-analyses. J Assist Reprod Genet 2020;37:459-69.

2. Kamel RM. Management of the infertile couple: an evidence-based protocol. Reprod Biol Endocrinol 2010;8:21.

3. Silva FR, Leite LD, Wassermann GF. Rapid signal transduction in Sertoli cells. Eur J Endocrinol 2002;147:425-33.

4. Ramaswamy S, Weinbauer GF. Endocrine control of spermatogenesis: role of FSH and LH/ testosterone. Spermatogenesis 2015;4: e996025.

5. Carnegie C. Diagnosis of hypogonadism: clinical assessments and laboratory tests. Rev Urol 2004;6 Suppl 6:S3-8.

6. Surampudi PN, Wang C, Swerdloff R. Hypogonadism in the aging male diagnosis, potential benefits, and risks of testosterone replacement therapy. Int J Endocrinol 2012;2012:625434.

7. Khera M, Broderick GA, Carson CC 3rd, Dobs AS, Faraday MM, Goldstein I, et al. Adult-onset hypogonadism. Mayo Clin Proc 2016;91:908-26.

8. Lo EM, Rodriguez KM, Pastuszak AW, Khera M. Alternatives to testosterone therapy: a review. Sex Med Rev 2018;6:106-13.

9. Lee JA, Ramasamy R. Indications for the use of human chorionic gonadotropic hormone for the management of infertility in hypogonadal men. Transl Androl Urol 2018;7(Suppl 3):S348-52.

10. Rohrmann S, Platz EA, Selvin E, Shiels MS, Joshu CE, Menke A, et al. The prevalence of low sex steroid hormone concentrations in men in the Third National Health and Nutrition Examination Sur- vey (NHANES III). Clin Endocrinol (Oxf) 2011;75:232-9.

11. World Health Organization. WHO laboratory manual for the examination and processing of human semen. Geneva: World Health Organization; 2021.

12. Krausz C, Hoefsloot L, Simoni M, Tuttelmann F, European Academy of Andrology; European Molecular Genetics Quality Network. $E A A / E M Q N$ best practice guidelines for molecular diagnosis of Y-chromosomal microdeletions: state-of-the-art 2013. Andrology 2014;2:5-19.

13. Liu PY, Baker HW, Jayadev V, Zacharin M, Conway AJ, Handelsman DJ. Induction of spermatogenesis and fertility during gonadotropin treatment of gonadotropin-deficient infertile men: predictors of fertility outcome. J Clin Endocrinol Metab 2009;94:801-8.

14. Whitten SJ, Nangia AK, Kolettis PN. Select patients with hypogonadotropic hypogonadism may respond to treatment with clomiphene citrate. Fertil Steril 2006;86:1664-8.

15. Baillargeon J, Urban RJ, Ottenbacher KJ, Pierson KS, Goodwin JS. Trends in androgen prescribing in the United States, 2001 to 2011. JAMA Intern Med 2013;173:1465-6.

16. Andrabi SW, Saini P, Joshi M, Mehta P, Makker GC, Mishra G, et al. HCG therapy in azoospermic men with lower or borderline testosterone levels and the prognostic value of $\mathrm{Y}$-deletion analysis in its outcome. Andrologia 2022;54:e14251. 